\title{
Correction: Generation of qualified clinical-grade functional hepatocytes from human embryonic stem cells in chemically defined conditions
}

Zhongwen Li, Jun Wu, Lei Wang, Weifang Han, Juan Yu, Xin Liu, Yukai Wang, Ying Zhang, Guihai Feng, Wei Li@, Glyn Nigel Stacey, Qi Gu, Baoyang Hu (D, Liu Wang, Qi Zhou (D) and Jie Hao

\section{Correction to: Cell Death and Disease}

https://doi.org/10.1038/s41419-019-1967-5

published online 10 October 2019
The original version of this article contained an error in the name of one of the co-authors (Glyn Nigel Stacey). This has been corrected in the PDF and HTML versions of the article.

Published online: 23 January 2020 